\title{
Prevalence and Associated Factors of Low Back Pain (LBP) among Adolescents in Central, Thailand
}

\author{
Boonsub Sakboonyarat ${ }^{1}$, Kritchaporn Chokcharoensap ${ }^{1}$, Monai Meesaeng ${ }^{1}$, Nattapong Jaisue ${ }^{1}$, \\ Dusit Janthayanont ${ }^{2} \&$ Phutsapong Srisawat $^{3}$ \\ ${ }^{1}$ Phramongkutklao College of Medicine, Bangkok, Thailand \\ ${ }^{2}$ Department of Family Medicine, Phramongkutklao College of Medicine, Bangkok, Thailand \\ ${ }^{3}$ Department of Orthopedics, Phramongkutklao College of Medicine, Bangkok, Thailand \\ Correspondence: Boonsub Sakboonyarat, Department of Military and Community Medicine, Phramongkutklao \\ College of Medicine, 315 Ratchawithi Rd Ratchathevi BKK 10400, Thailand. Tel: 66-85-95-45955. E-mail: \\ boonsub1991@pcm.ac.th, countryside.physician@gmail.com
}

Received: November 9, 2017 Accepted: December 14, 2017 Online Published: December 20, 2017

doi:10.5539/gjhs.v10n2p49 URL: https://doi.org/10.5539/gjhs.v10n2p49

\begin{abstract}
Background: Low Back Pain (LBP) is one of the most common musculoskeletal disorders found from adolescent to the elderly. These affect the quality of life of adolescents due to the inability to fully perform any activity. The information concerning LBP in Thailand is still limited especially among adolescents. Most related studies were conducted among young adult. The present study was aimed to determine the prevalence and the associated factors of LBP among adolescents in Central Thailand.
\end{abstract}

Methods: A total of 4944 adolescents were included in the study. The Nordic Standard Questionnaire (NSQ) was used to screen LBP conditions among these adolescents. Standardized questionnaires were used to collect demographic data and associated factors.

Results: The prevalence of LBP among adolescents was $26.7 \%$. Univariate and multivariate analysis showed that adolescents with LBP were associated with both behavioral and physical activity factors. These factors included sex, grade, using a smartphone, transportation to school, suitability of chairs and desks, history of back injury and low frequency of exercise

Conclusion: Our data emphasized that LBP was a problem among adolescent. Supportive exercise and physical activity should be provided for adolescents.

Keywords: Low Back Pain, LBP, prevalence, associated factors, adolescents, Thailand

\section{Introduction}

Low Back Pain (LBP) is one of the most common musculoskeletal disorders found from adolescents to the elderly. Most cases of LBP are due to nonspecific causes; however, the role these risk factors play remains controversial. The period prevalence at 12 months of LBP among adolescents was between 17.4 to $60.3 \%$ in differing demographic areas (Calvo, Gómez, \& Sánchez, 2013); In Thailand, a related study among 684 healthy Thai university students was conducted in 2015; a total of 524 (77\%) students were followed for 1 year. In all, $31 \%$ reported a new onset of LBP (Kanchanomai, Janwantanakul, Pensri, \& Jiamjarasrangsi, 2015). Several personal-related factors including age, height, body mass index (BMI), were shown to be associated with LBP symptoms. Other factors such as daily and physical activity were also shown to be significantly associated with LBP (Louw, Morris, \& Grimmer, 2007; Silva, Badaró \& Dall'Agnol, 2014; Shan et al., 2013). Unresolved LBP of adolescents could lead to chronic back pain. This affects the quality of life of these adolescents (Wilson, Eriksson, D'Eon, Mikail, \& Emery, 2002). For example, limiting movement of any part of body would result in the inability to fully perform any activity. Also, when these adolescents could not resolve or modify any risk factors, it could lead to long term problems (McGorry, BSPT, Snook, \& Hsiang, 2000). Adolescence is the period in human growth and development occurring after childhood and before adulthood, from ages 10 to 19. Adolescents differ from both young children and adults regarding areas as growth and development, physical activity and behavior. The information on LBP in Thailand is still limited especially among adolescents. Most related studies were conducted among young adults. The aims of this study were to determine the prevalence and associated factors of LBP among 
adolescents in central Thailand.

\section{Method}

The present study was conducted in a provincial secondary school in PhraNakhon Si Ayutthaya, central Thailand. A cross-sectional study identified the prevalence and associated factors of LBP among adolescents. A total survey was conducted among 4,944 students from November to December 2014.

\subsection{Study populations}

The present study was conducted in a provincial secondary school in PhraNakhon Si Ayutthaya, central Thailand.

\subsection{Participant Characteristics}

2.2.1 Inclusion criteria

- Adolescents who had 11 to 19 years old in provincial secondary school in PhraNakhon Si Ayutthaya in November 2014.

- Adolescents whose parents signed consent forms.

\subsubsection{Exclusion criteria}

- Adolescents reporting musculoskeletal problems such as fractures in the upper limbs, lower limbs and trunk, having a prosthesis and spinal disease were excluded.

\section{Measures and Covariates}

Primary outcomes

1. Prevalence of low back pain among adolescents

2. Associated factors of low back pain

\subsection{Quantitative study}

\subsubsection{Sample Size Calculation}

A related study on low back pain among adolescents in Shanghai (Shan et al., 2013), reported a prevalence of $33.1 \%$. The sample size calculated with infinite population was

$$
n=\frac{z^{2}(P)(1-P)}{d^{2}}
$$

$\mathrm{n}=$ Sample size

$\mathrm{Z}=\mathrm{Z}$-value $=1.96$

$\mathrm{P}=$ Percentage of population selecting a choice $=0.33$

$\mathrm{d}=$ Confident interval $=5 \%$ of $\mathrm{P}=0.0165$

Alpha $(\alpha)=0.05$

Therefore, the sample size (n) was 3,120 participants

\subsubsection{Sampling Procedures}

Considering the large sample size, a total survey was conducted in the area. The Nordic Standard Questionnaire (NSQ) (Kuorinka et al., 1987) was used to screen LBP conditions among these adolescents. The NSQ consists of special questions to assess low back pain symptoms. These questionnaires cover the duration of the symptoms including entire life, last 12 months and previous 7 days. Adolescents reporting LBP symptoms, i.e., ache, pain or discomfort in the lower back area whether or not it extended from there or to one or both legs since 12 months ago would be identified as having LBP.

To determine the associated factors of LBP among these adolescents, face-to-face interviews using standardized questionnaires were conducted. The questionnaires covered demographic information, physical activity, smartphone and tablet use, exercise, the suitability of chairs and desks in the classroom and transportation to school.

Collected data were checked for accuracy and completeness. Then the data were coded and entered into the software packages for computer (SPSS for Window, version 23). Demographic data and prevalence was analyzed using descriptive statistics. Binary logistic regression analysis was used to determine the associated factors of LBP. The magnitude of association was presented as crude odds ratios (ORs) and adjusted odds ratios (AORs) was 
presented as $95 \%$ confidence interval. A $p$-value of less than 0.05 was considered as statistically significant.

\subsection{Ethical Considerations}

This study was reviewed and approved by the Institutional Review Board, Phra Nakhon Sri Ayutthaya Hospital under the Ministry of Public Health. Informed consent was obtained from the main guardian of each adolescent.

\subsection{Operational Definitions and Abbreviations}

LBP $=$ low back pain

$\mathrm{BMI}=$ body mass index

NSQ $=$ Nordic standard questionnaire

\section{Results}

Of 4,944 adolescents, 4190 (84.8\%) were included in this study and 4,162 (84.2\%) were confirmed as meeting the criteria. Data analysis was based on the validated questionnaires. Demographic data of these adolescents are shown in Table 1. The participants had a higher proportion of males than females ( $58.8 \%$ vs. $41.2 \%$, respectively). Most adolescents were 14 to 16 years old (54.8\%). An estimated $44.2 \%$ of participants had a normal range of BMI $\left(18.5 \mathrm{~kg} / \mathrm{m}^{2}-24.9 \mathrm{~kg} / \mathrm{m}^{2}\right)$.

Using the NSQs, the overall prevalence of LBP in this population was $26.7 \%$; the prevalence of LBP in each subgroup is shown in Table 2. Univariate and multivariate analysis were performed to identify the associated factors of LBP. The associated factors of LBP included both behavioral and physical activity factors. Females had a significantly higher prevalence of LBP than males $(\mathrm{OR}=1.40,95 \% \mathrm{CI} 1.20-1.63)$. The prevalence of LBP among these adolescents tended to be higher with grade level ( $p$-value $<0.05)$. Among all participants, $94.4 \%(3,928$ of $4,162)$ were smartphone users and 1,071 of $3,928(27.3 \%)$ smartphone users had significant LBP (OR=1.66, $95 \% \mathrm{CI} 1.17-2.36)$. Most adolescents went to school by bus, car and motorcycle, by rank. The adolescents using bus and motorcycle revealed a higher prevalence of LBP than those transported by car $(\mathrm{OR}=1.21,95 \% \mathrm{CI}$ 1.03-1.43 and $\mathrm{OR}=1.39,95 \% \mathrm{CI} 1.13-1.72$ ). Uncomfortable seats and desks were associated with LBP among adolescents $(\mathrm{OR}=1.51,95 \% \mathrm{CI} 1.27-1.79$ and $\mathrm{OR}=1.29,95 \% \mathrm{CI} 1.02-1.62)$. Adolescents reporting a history of back injury were significantly associated with $\mathrm{LBP}$ with $\mathrm{OR}=2.11,95 \% \mathrm{CI} 1.75-2.55$. The frequency of exercise (two to seven times weekly) indicated the protective effect of $\operatorname{LBP}(p$-value $<0.05)$.

Table 1. Characteristics of the participants and prevalence of low back pain in adolescents

\begin{tabular}{lcc}
\hline \multirow{2}{*}{ Characteristics } & Total & Low back pain \\
\cline { 2 - 3 } & n (\%) & $\mathbf{n}(\%)$ \\
\hline Gender & & \\
Fale & $2445(58.75)$ & $571(23.40)$ \\
Age (yrs.) & $1717(41.25)$ & $542(31.60)$ \\
$11-13$ & mean (SD) & $14.86(1.68)$, min-max $=12-19$ \\
$14-16$ & $1025(24.63)$ & $193(18.80)$ \\
$17-19$ & $2280(54.78)$ & $617(27.10)$ \\
Grade & $857(20.59)$ & $303(35.40)$ \\
$7^{\text {th }}$ & & $131(16.70)$ \\
$8^{\text {th }}$ & $785(18.86)$ & $214(26.40)$ \\
$9^{\text {th }}$ & $811(19.49)$ & $198(22.80)$ \\
$10^{\text {th }}$ & $868(20.85)$ & $192(32.20)$ \\
$11^{\text {th }}$ & $596(14.32)$ & $187(34.10)$ \\
$12^{\text {th }}$ & $549(13.19)$ & $191(34.50)$ \\
\hline
\end{tabular}




\begin{tabular}{|c|c|c|}
\hline Height (cm) & \multicolumn{2}{|c|}{ mean $(S D)=163.04(8.40), \min -\max =110-189$} \\
\hline$<141$ & $23(0.55)$ & $8(36.40)$ \\
\hline $141-150$ & $227(5.45)$ & $55(24.20)$ \\
\hline $151-160$ & $1452(34.89)$ & $398(27.40)$ \\
\hline $161-170$ & $1667(40.05)$ & $427(25.60)$ \\
\hline$>170$ & 793 (19.06) & $224(28.20)$ \\
\hline BMI $\left(\mathrm{kg} / \mathrm{m}^{2}\right)$ & \multicolumn{2}{|c|}{ mean $(S D)=20.89(4.74)$, min- $\max =11.2-56.2$} \\
\hline$<18.5$ & $1642(39.45)$ & $441(26.90)$ \\
\hline $18.5-22.9$ & $364(8.75)$ & $118(32.40)$ \\
\hline $23-24.9$ & $1477(35.49)$ & $363(24.60)$ \\
\hline $25-29.9$ & $452(10.86)$ & $132(29.20)$ \\
\hline$>30$ & $227(5.45)$ & $58(25.60)$ \\
\hline \multicolumn{3}{|c|}{ Smartphone used } \\
\hline No & $234(5.62)$ & $42(17.9)$ \\
\hline Yes & $3928(94.38)$ & $1071(27.30)$ \\
\hline \multicolumn{3}{|l|}{ Tablet used } \\
\hline No & $2856(68.62)$ & $754(26.40)$ \\
\hline Yes & $1306(31.38)$ & $359(27.50)$ \\
\hline \multicolumn{3}{|c|}{ Transportation to school } \\
\hline By car & $1448(34.79)$ & $348(24.00)$ \\
\hline By bus & $1904(45.75)$ & $520(27.30)$ \\
\hline By motorcycle & $693(16.65)$ & $204(29.40)$ \\
\hline By bicycle & $18(0.43)$ & $7(38.90)$ \\
\hline On foot & $99(2.38)$ & $34(34.30)$ \\
\hline
\end{tabular}

Table 2. Sociodemographic factors, physical activity and the associated factors of LBP in adolescents

\begin{tabular}{|c|c|c|c|c|c|}
\hline \multirow{2}{*}{ Factors } & \multirow{2}{*}{$\begin{array}{l}\text { Total } \\
\text { n (\%) }\end{array}$} & \multirow{2}{*}{$\begin{array}{l}\text { LBP } \\
\text { n (\%) }\end{array}$} & \multirow{2}{*}{ Crude OR } & \multirow{2}{*}{$(95 \% \mathrm{CI})$} & \multirow{2}{*}{$p$-value } \\
\hline & & & & & \\
\hline \multicolumn{6}{|l|}{ Gender } \\
\hline Male & $2445(58.75)$ & $571(23.40)$ & 1 & & \\
\hline Female & $1717(41.25)$ & $542(31.60)$ & 1.51 & $(1.32-1.74)$ & $<0.001$ \\
\hline \multicolumn{6}{|l|}{ Age (yrs.) } \\
\hline $11-13$ & $1025(24.63)$ & $193(18.80)$ & 1 & & \\
\hline $14-16$ & $2280(54.78)$ & $617(27.10)$ & 1.60 & $(1.33-1.92)$ & $<0.001$ \\
\hline $17-19$ & 857 (20.59) & $303(35.40)$ & 2.36 & $(1.91-2.91)$ & $<0.001$ \\
\hline \multicolumn{6}{|l|}{ Grade } \\
\hline $7^{\text {th }}$ & $785(18.86)$ & $131(16.70)$ & 1 & & \\
\hline $8^{\text {th }}$ & 811 (19.49) & $214(26.40)$ & 1.79 & $(1.40-2.28)$ & $<0.001$ \\
\hline $9^{\text {th }}$ & $868(20.85)$ & $198(22.80)$ & 1.48 & $(1.15-1.89)$ & 0.002 \\
\hline $10^{\text {th }}$ & $596(14.32)$ & $192(32.20)$ & 2.37 & $(1.84-3.06)$ & $<0.001$ \\
\hline $11^{\text {th }}$ & 549 (13.19) & $187(34.10)$ & 2.58 & $(1.99-3.34)$ & $<0.001$ \\
\hline $12^{\text {th }}$ & $553(13.30)$ & $191(34.50)$ & 2.63 & $(2.04-3.41)$ & $<0.001$ \\
\hline
\end{tabular}




\section{Height (cm)}

$\begin{array}{ll}<141 & 23(0.55) \\ 141-150 & 227(5.45) \\ 151-160 & 1452(34.89) \\ 161-170 & 1667(40.05) \\ >170 & 793(19.06)\end{array}$

BMI $\left(\mathrm{kg} / \mathrm{m}^{2}\right)$

$\begin{array}{ll}<18.5 & 1642(39.45) \\ 18.5-22.9 & 364(8.75) \\ 23-24.9 & 1477(35.49) \\ 25-29.9 & 452(10.86) \\ >30 & 227(5.45)\end{array}$

Frequency of weekly exercise (times)

$\begin{array}{ll}\leq 1 & 386(9.30) \\ 2-4 & 2729(65.57) \\ 5-7 & 714(17.16) \\ >7 & 333(8.00)\end{array}$

Average time of each exercise (hours)

$\begin{array}{ll}<0.5 & 1438(34.55) \\ 0.5-1 & 2144(51.51) \\ 1-2 & 446(10.72) \\ >2 & 134(3.22)\end{array}$

Intensity of regular physical activities

$\begin{array}{ll}\text { Light } & 1115(26.79) \\ \text { Moderate } & 2672(64.20) \\ \text { Heavy } & 375(9.01)\end{array}$

$\begin{array}{llll}8(36.40) & 1 & & \\ 55(24.20) & 0.56 & (0.22-1.40) & 0.216 \\ 398(27.40) & 0.66 & (0.25-1.59) & 0.357 \\ 427(25.60) & 0.60 & (0.26-1.67) & 0.257 \\ 224(28.20) & 0.69 & & 0.408\end{array}$

$441(26.90) \quad 1$

$118(32.40) \quad 1.13$

$363(24.60) \quad 1.47$

$(0.96-1.33) \quad 0.002$

$132(29.20) \quad 1.27$

$(1.15-1.89) \quad 0.034$

$58(25.60) \quad 1.05$

$(1.00-1.60) \quad 0.322$

$(0.76-1.45) \quad 0.076$

$\begin{array}{llll}145(37.60) & 1 & & \\ 715(26.20) & 0.59 & (0.47-0.74) & 0.14 \\ 163(22.80) & 0.49 & (0.38-0.64) & 0.746 \\ 90(27.00) & 0.62 & (0.45-0.85) & 0.003\end{array}$

$\begin{array}{llll}355(24.70) & 1 & & \\ 587(27.40) & 1.15 & (0.99-1.34) & 0.073 \\ 135(30.30) & 1.32 & (1.05-1.68) & 0.019 \\ 36(26.90) & 1.12 & (0.75-1.67) & 0.577\end{array}$

Table 3. Smartphone and tablet use and the associated factors of LBP in adolescents

\begin{tabular}{|c|c|c|c|c|c|}
\hline \multirow{2}{*}{ Factors } & Total & LBP & \multirow{2}{*}{ Crude OR } & \multirow{2}{*}{$(95 \% \mathrm{CI})$} & \multirow{2}{*}{$p$-value } \\
\hline & n (\%) & n (\%) & & & \\
\hline \multicolumn{6}{|c|}{ Smartphone used } \\
\hline No & $234(5.62)$ & $42(17.90)$ & 1 & & \\
\hline Yes & $3928(94.38)$ & $1071(27.30)$ & 1.71 & $(0.43-0.82)$ & 0.002 \\
\hline \multicolumn{6}{|c|}{ Operating system of smartphone. } \\
\hline ios Apple & $1524(38.86)$ & $443(29.10)$ & 1 & & \\
\hline Window & $170(4.34)$ & $47(27.60)$ & 0.93 & $(0.66-1.33)$ & 0.698 \\
\hline Android & $2123(54.16)$ & $547(25.80)$ & 0.85 & $(0.73-0.99)$ & 0.303 \\
\hline Blackberry & $64(1.63)$ & $18(28.10)$ & 0.96 & $(0.55-1.67)$ & 0.871 \\
\hline other & $40(1.03)$ & $13(32.50)$ & 1.18 & $(0.60-2.30)$ & 0.638 \\
\hline \multicolumn{6}{|c|}{ Size of the smartphone screen (inch) } \\
\hline$\leq 4.7$ & $2754(73.28)$ & $771(28.00)$ & 1 & & \\
\hline $4.7-5.7$ & $873(23.23)$ & $236(27.00)$ & 1.20 & $(0.80-1.81)$ & 0.580 \\
\hline$>5.7$ & $131(3.49)$ & $32(24.40)$ & 1.15 & $(0.55-1.2)$ & 0.374 \\
\hline
\end{tabular}


Total time spent on smartphone (months)

$\begin{array}{llllll}<6 & 928(23.63) & 221(23.80) & 1 & & \\ \geq 6 & 3000(76.37) & 850(28.30) & 1.27 & (1.07-1.50) & 0.007\end{array}$

Average time spent on smartphone daily (hours)

$\begin{array}{llllll}<0.5 & 378(9.65) & 92(24.30) & 1 & & \\ 0.5-1.5 & 1445(36.88) & 358(24.80) & 1.02 & (0.79-1.33) & 0.861 \\ >1.5 & 2095(53.47) & 618(29.50) & 1.30 & (1.01-1.68) & 0.042\end{array}$

Eye-to-screen distance while using smartphone $(\mathrm{cm})$

$\begin{array}{llllll}\leq 15 & 1968(50.27) & 534(27.10) & 1 & & \\ >15 & 1947(49.73) & 534(27.40) & 1.02 & (0.88-1.17) & 0.837\end{array}$

Tablet used

$\begin{array}{llllll}\text { No } & 2856(68.62) & 754(26.40) & 1 & & \\ \text { Yes } & 1306(31.38) & 359(27.50) & 1.06 & (0.91-1.22) & 0.462\end{array}$

Total time spent on tablet (months)

$\begin{array}{ll}<6 & 928(23.63) \\ \geq 6 & 3000(76.37)\end{array}$

$\begin{array}{ll}221(23.80) & 1 \\ 850(28.30) & 1.13\end{array}$

Average time spent on tablet daily (hours)

$\begin{array}{llllll}<0.5 & 297(29.64) & 82(27.60) & 1 & & \\ 0.5-1.5 & 454(45.31) & 114(25.10) & 0.88 & (0.63-1.22) & 0.446 \\ >1.5 & 369(36.83) & 118(32.00) & 1.23 & (0.88-1.72) & 0.222\end{array}$

Eye-to-screen distance while using tablet $(\mathrm{cm})$

$\begin{array}{llllll}\leq 15 & 527(47.10) & 143(27.10) & 1 & & \\ >15 & 592(52.90) & 169(28.50) & 1.07 & (0.83-1.39) & 0.599\end{array}$

Table 4. Daily life activities and associated factors of LBP in adolescents

\begin{tabular}{|c|c|c|c|c|c|}
\hline \multirow{2}{*}{ Factors } & \multirow{2}{*}{$\begin{array}{c}\text { Total } \\
\text { n (\%) }\end{array}$} & \multirow{2}{*}{$\begin{array}{l}\text { LBP } \\
\text { n (\%) }\end{array}$} & \multirow{2}{*}{ Crude OR } & \multirow{2}{*}{$(95 \% \mathrm{CI})$} & \multirow{2}{*}{ p-value } \\
\hline & & & & & \\
\hline \multicolumn{6}{|c|}{ Transportation to school } \\
\hline By car & $1448(34.79)$ & $348(24.00)$ & 1 & & \\
\hline By bus & $1904(45.75)$ & $520(27.30)$ & 1.19 & $(1.02-1.39)$ & 0.032 \\
\hline By motorcycle & $693(16.65)$ & $204(29.40)$ & 1.32 & $(1.08-1.62)$ & 0.008 \\
\hline By bicycle & $18(0.43)$ & $7(38.90)$ & 2.01 & $(0.77-5.23)$ & 0.152 \\
\hline On foot & $99(2.38)$ & $34(34.30)$ & 1.65 & $(1.07-2.55)$ & 0.023 \\
\hline \multicolumn{6}{|c|}{ Averager bag weight (kg) } \\
\hline$<2$ & $2860(68.72)$ & $716(25.00)$ & 1 & & \\
\hline$\geq 2$ & $1302(31.28)$ & $397(30.5 .0)$ & 1.31 & $(1.14-1.52)$ & $<0.001$ \\
\hline \multicolumn{6}{|l|}{ Seat in class } \\
\hline Convenient & $3214(77.22)$ & $767(23.90)$ & 1 & & \\
\hline Inconvenient & $948(22.78)$ & $346(36.50)$ & 1.83 & $(1.57-2.14)$ & $<0.001$ \\
\hline \multicolumn{6}{|l|}{ Desk height } \\
\hline Convenient & $3746(90.00)$ & $956(25.50)$ & 1 & & \\
\hline Inconvenient & $416(10.00)$ & $157(37.70)$ & 1.77 & $(1.43-2.19)$ & $<0.001$ \\
\hline
\end{tabular}




\section{Chair back support}

Yes

No

4074 (97.89)

$88(2.11)$

Time per class (hours)

$\begin{array}{ll}1 & 700(16.82) \\ 2 & 117(2.81) \\ 3 & 1986(47.71) \\ \geq 4 & 1359(32.66)\end{array}$

Posture while watching TV

$\begin{array}{ll}\text { Lying } & 1219(29.29) \\ \text { Sitting } & 899(21.60) \\ \text { Semi reclining } & 2044(49.11)\end{array}$

Average time spent on TV daily (hours)

$\begin{array}{ll}<1 & 1228(31.27) \\ 1-4 & 1543(39.29) \\ >4 & 1156(29.44)\end{array}$

History of accident related LBP

$\begin{array}{lrr}\text { No } & 3564(85.63) & 878(24.60) \\ \text { Yes } & 598(14.37) & 235(39.30)\end{array}$

Table 5. Multivariate analysis for factors associated with LBP in adolescents

\begin{tabular}{|c|c|c|c|c|c|}
\hline \multirow{2}{*}{ Factors } & Total & LBP & \multirow{2}{*}{ Adjusted O.R. } & \multirow{2}{*}{$(95 \% \mathrm{CI})$} & \multirow{2}{*}{$p$-value } \\
\hline & n (\%) & n (\%) & & & \\
\hline \multicolumn{6}{|l|}{ Gender } \\
\hline Male & $2445(58.75)$ & $571(23.40)$ & 1 & & \\
\hline Female & $1717(41.25)$ & $542(31.60)$ & 1.40 & $(1.20-1.63)$ & $<0.001$ \\
\hline \multicolumn{6}{|l|}{ Grade } \\
\hline $7^{\text {th }}$ & $785(18.86)$ & $131(16.70)$ & 1 & & \\
\hline $8^{\text {th }}$ & $811(19.49)$ & $214(26.40)$ & 1.77 & $(1.38-2.26)$ & $<0.001$ \\
\hline $9^{\text {th }}$ & $868(20.85)$ & $198(22.80)$ & 1.42 & $(1.11-1.83)$ & 0.006 \\
\hline $10^{\text {th }}$ & $596(14.32)$ & $192(32.20)$ & 2.21 & $(1.70-2.86)$ & $<0.001$ \\
\hline $11^{\text {th }}$ & $549(13.19)$ & $187(34.10)$ & 2.30 & $(1.77-3.00)$ & $<0.001$ \\
\hline $12^{\text {th }}$ & $553(13.30)$ & $191(34.50)$ & 2.33 & $(1.78-3.04)$ & $<0.001$ \\
\hline \multicolumn{6}{|c|}{ Frequency of weekly exercise (times) } \\
\hline$\leq 1$ & $386(9.30)$ & $145(37.60)$ & 1 & & \\
\hline $2-4$ & $2729(65.57)$ & $715(26.20)$ & 0.67 & $(0.53-0.84)$ & 0.001 \\
\hline $5-7$ & $714(17.16)$ & $163(22.80)$ & 0.62 & $(0.47-0.83)$ & 0.001 \\
\hline$>7$ & $333(8.00)$ & $90(27.00)$ & 0.77 & $(0.55-1.08)$ & 0.134 \\
\hline \multicolumn{6}{|c|}{ Smartphone used } \\
\hline No & $234(5.62)$ & $42(17.9)$ & 1 & & \\
\hline Yes & 3928 (94.38) & $1071(27.30)$ & 1.66 & $(1.17-2.36)$ & 0.005 \\
\hline
\end{tabular}




\begin{tabular}{llllll}
\multicolumn{5}{l}{ Transportation to school } \\
By car & $1448(34.79)$ & $348(24.00)$ & 1 & & \\
By bus & $1904(45.75)$ & $520(27.30)$ & 1.21 & $(1.03-1.43)$ & 0.021 \\
By motorcycle & $693(16.65)$ & $204(29.40)$ & 1.39 & $(1.13-1.72)$ & 0.002 \\
By bicycle & $18(0.43)$ & $7(38.90)$ & 2.10 & $(0.78-5.67)$ & 0.142 \\
On foot & $99(2.38)$ & $34(34.30)$ & 1.57 & $(1.00-2.47)$ & 0.05 \\
Seat in class & & & & & \\
Convenient & $3214(77.22)$ & $767(23.90)$ & 1 & $(1.27-1.79)$ & $<0.001$ \\
Inconvenient & $948(22.78)$ & $346(36.50)$ & 1.51 & & \\
Desk height & & & & & \\
Convenient & $3746(90.00)$ & $956(25.50)$ & 1 & & \\
Inconvenient & $416(10.00)$ & $157(37.70)$ & 1.29 & & \\
History of accident related LBP & & & & \\
No & $3564(85.63)$ & $878(24.60)$ & 1 & & \\
\multicolumn{2}{l}{ Yes } & $598(14.37)$ & $235(39.30)$ & 2.11 & \\
\hline
\end{tabular}

Multivariate logistic regression (Backward wald) Adjusted for: Gender, Grade, Frequency of weekly exercise, Smartphone use, Transportation to school, Seat in class, Desk height, History of accident relate LBP.

\section{Discussion}

The prevalence of LBP in the present study was $26.7 \%$, similar to a related study in Thailand (Kanchanomai et al., 2015). A recent study conducted among 684 healthy Thai university students to determine the prevalence of LBP among undergraduate students enrolled a total of 524 (77\%) students and followed up for one year. A total of $31 \%$ reported a new onset of LBP. The prevalence of LBP in the present study was the same as a recent study from China that also showed the prevalence of LBP among adolescents living in Shanghai, was 33.1\% (Shan et al., 2013). One of the limitations in this study was that only adolescents from one school were included. However, this school was a provincial secondary school, so these adolescents came from different districts. It constitutes the biggest secondary school in the province, in central Thailand, so the participants could be representative of adolescents throughout Thailand.

Most studies found that LBP was common among females (Shan et al., 2013; Fernandes et al., 2015; Troussier, Davoine, de Gaudemaris, Fauconnier \& Phelip, 1994; Balagué et al., 1994; Diepenmaat, 2006). Our finding showed that the prevalence of LBP among females was significantly higher than males. LBP was common among females probably related to female hormonal change and the menstrual cycle (Balagué et al., 1994; Wedderkopp, Andersen, Froberg \& Leboeuf-Yde, 2005). In addition, the physical strength of females was lower than males, putting females increasingly at risk of musculoskeletal overload (Fernandes et al., 2015). In addition, most females tended to have more mental stress than males, whereas stress has been found to correlate with musculoskeletal disease (Shan et al., 2013; Mikkelsson, Salminen \& Kautiainen, 1997; Härmä, Kaltiala-Heino, Rimpelä \& Rantanen, 2002) and male had higher pain thresholds than females (Shan et al., 2013; Torgén \& Swerup, 2002).

One related study indicated that adolescents with LBP tended to be from upper grades and a few studies have revealed that LBP correlated with increased age (Shan et al., 2013; Fernandes et al., 2015; Sato et al., 2008; Olsen T et al., 1992; Salminen, Pentti, \& Terho, 1992). Our study also revealed that the prevalence of LBP increased in upper grades in secondary school. This result might be related to adolescents having enhanced growth and consequent decreased flexibility, including that of the hamstring and the quadriceps muscles, causing functional failure of the lumbar muscles as a result of back pain (Fernandes et al., 2015; Poussa et al., 2005). In addition this result might be associated with the academic and psychological pressure placed on upper grade high school students due to frequent examinations such as the Ordinary National Education Test (O-net), and the university entrance examination. It may also the increase stress and sedentary states and reduce physical activity (Shan et al., 2013).

The adolescents who used smartphones were significantly associated with LBP. This associated factor could 
possibly have been caused by adolescents using their smartphone to play games with their friends. The body posture adopted while they used the smartphone involved sitting on the floor and bending their backs forward, so this poor posture could have caused musculoskeletal overload and consequently, pain. One recent study from China also showed that high school students used mobile phone showed a significantly high prevalence of LBP. This was probably because mobile phone users establish a comfortable posture, and sometimes have to remain in static postures while using the phone (Shan et al., 2013). This static posture can increase bone and muscle stress around the waist and is closely related to LBP (Yue, Liu, \& Li, 2012).

In the present study, the adolescents who went to school by bus and motorcycle tended to present a higher prevalence of LBP than adolescents who went by car. Most adolescents, who take the bus, might have to carry their baggage and stand for a long time. As a result, their lumbar and back muscles would become overloaded and cause muscle strain. A few adolescents went to school by motorcycle and sat on the saddle and carried their baggage. Those who bent their backs and leaned their lumbar muscles forward sometimes had to remain in a static posture resulting in LBP.

In our study, uncomfortable seating constituted a risk effect. Our study agreed with a reported study in Brazil (Onofrio, da Silva, Domingues, \& Rombaldi, 2011), where uncomfortable seating was associated with a higher prevalence of LBP. Using seats, which are too narrow or too small, could result in limits to both the postural changes and relaxation parts of the extensor musculature, indicating increased back discomfort (Salewytsch \& Callaghan, 1999). Using uncomfortable desks was associated with low back pain. Recent studies from Saudi Arabia also revealed that too low or too high table heights relative to a student's body dimensions increased the stress at the lumbosacral area resulting in discomfort (Ramadan, 2011).

In the present study, adolescents having a history of back injury were significantly associated with LBP as well as those in the study by (Troussier et al., 1994) with odds ratio of 5.50 (2.97- 0.98). Adolescence constitutes an age involving much physical activity, e.g., playing sports, driving, traveling and involving extreme or adventurous behaviors. This may result in accidents and injuries to the back. A recent study revealed that moderate or severe low back pain was common among patient who experienced motor vehicle collisions. These events might lead to sacroiliac and facet joint pain (Bortsov, 2015; DePalma, 2011).

The frequency of exercise was significantly associated with LBP among adolescents. This study also revealed that frequency of exercise, more than once weekly, created a protective effect of LBP as well as a related study conducted among adolescents aged 15 years. They found a correlation between low frequency of physical activity, less than or equal to twice weekly, and LBP (McGorry, 2000; Salminen, Oksanen, Mäki, Pentti \& Kujala, 1993). This result might be described by low frequency of exercise or physical activity, resulting in decreased endurance of the abdominal and lumbar muscles (Salminen, Erkintalo, Laine, \& Pentti, 1995). One recent study indicated that regular physical activity improved fitness and did not increase the risk of LBP (Ribaud; 2013). Consequently, teachers should provide and encourage exercise for students at least twice weekly to improve the strength of any musculature including the lumbar, abdominal and all extremity muscles.

In conclusion, we present a high prevalence of LBP among adolescents in central, Thailand. LBP among adolescents was associated with both behavioral and physical activity factors. Our information is crucial for schools and parents to decrease the risk of the LBP among adolescents.

\section{Acknowledgments}

We thank the staff at the Department of Orthopedics and Family Medicine, Phramongkutklao Hospital and the College of Medicine, for their support in completing this study. We thank the teachers and students at Ayutthaya Witthayalai School, PhraNakhon Si Ayutthaya, central Thailand, for their cooperation in collecting data for this article. We thank Professor Colonel Mathirut Mungthin for support and for proofreading this manuscript.

\section{Competing Interests Statement}

The authors declare that they have no competing or potential conflicts of interest.

\section{References}

Adams, M. A., Mcnally, D. S., \& Dolan, P. (1997). Stress Distributions Inside Intervertebral Discs. The Journal of Bone and Joint Surgery-british Volume, 79(3S), 283. https://doi.org/10.1302/0301-620x78b6.1287

Balague, F., Nordin, M., Skovron, M. L., Dutoit, G., Yee, A., \& Waldburger, M. (1994). Non-specific low-back pain among schoolchildren: a field survey with analysis of some associated factors. Clinical Spine Surgery, 7(5), 374-379. https://doi.org/10.1097/00002517-199410000-00002

Bortsov, A. V., Platts-Mills, T. F., Peak, D. A., Jones, J. S., Swor, R. A., Domeier, R. M., ... McLean, S. A. 
(2014). Effect of pain location and duration on life function in the year after motor vehicle collision. PAIN®, 155(9), 1836-1845. https://doi.org/10.1016/j.pain.2014.06.013

Calvo-Muñoz, I., Gómez-Conesa, A., \& Sánchez-Meca, J. (2013). Prevalence of low back pain in children and adolescents: a meta-analysis. BMC pediatrics, 13(1), 14. https://doi.org/10.1186/1471-2431-13-14

DePalma, M., Ketchum, J., Saullo, T., \& Schofferman, J. (2011). Structural etiology of chronic low back pain due to motor vehicle collision. Pain medicine, 12(11), 1622-1627. https://doi.org/10.1111/j.1526-4637.2011.01246.x

Diepenmaat, A. C. M., Van der Wal, M. F., De Vet, H. C. W., \& Hirasing, R. A. (2006). Neck/shoulder, low back, and arm pain in relation to computer use, physical activity, stress, and depression among Dutch adolescents. Pediatrics, 117(2), 412-416. https://doi.org/10.1542/peds.2004-2766

Fernandes, J. A. A., Genebra, C. V. D. S., Maciel, N. M., Fiorelli, A., Conti, M. H. S. D., \& De Vitta, A. (2015). Low back pain in schoolchildren: a cross-sectional study in a western city of São Paulo State, Brazil. Acta ortopedica brasileira, 23(5), 235-238. https://doi.org/10.1590/1413-785220152305148842

Härmä, A. M., Kaltiala-Heino, R., Rimpelä, M., \& Rantanen, P. (2002). Are adolescents with frequent pain symptoms more depressed?. Scandinavian journal of primary health care, 20(2), 92-96. https://doi.org/10.1080/713796398

Kanchanomai, S., Janwantanakul, P., Pensri, P., \& Jiamjarasrangsi, W. (2015). A prospective study of incidence and risk factors for the onset and persistence of low back pain in Thai university students. Asia Pacific Journal of Public Health, 27(2), NP106-NP115. https://doi.org/10.1177/1010539511427579

Kuorinka, I., Jonsson, B., Kilbom, A., Vinterberg, H., Biering-Sørensen, F., Andersson, G., \& Jørgensen, K. (1987). Standardised Nordic questionnaires for the analysis of musculoskeletal symptoms. Applied ergonomics, 18(3), 233-237. https://doi.org/ 10.1016/0003-6870(87)90010-x

Louw, Q. A., Morris, L. D., \& Grimmer-Somers, K. (2007). The prevalence of low back pain in Africa: a systematic review. BMC Musculoskeletal disorders, 8(1), 105. https://doi.org/10.1186/1471-2474-8-105

McGorry, R. W., Bspt, B. S. W., Snook, S. H., \& Hsiang, S. M. (2000). The relation between pain intensity, disability, and the episodic nature of chronic and recurrent low back pain. Spine, 25(7), 834-841. https://doi.org/10.1097/00007632-200004010-00012

Mikkelsson, M., Salminen, J. J., \& Kautiainen, H. (1997). Non-specific musculoskeletal pain in preadolescents. Prevalence and 1-year persistence. Pain, 73(1), 29-35. https://doi.org/10.1016/s0304-3959(97)00073-0.

Olsen, T. L., Anderson, R. L., Dearwater, S. R., Kriska, A. M., Cauley, J. A., Aaron, D. J., \& LaPorte, R. E. (1992). The epidemiology of low back pain in an adolescent population. American journal of public health, 82(4), 606-608. https://doi.org/10.2105/ajph.82.4.606

Onofrio, A. C., Da Silva, M. C., Domingues, M. R., \& Rombaldi, A. J. (2012). Acute low back pain in high school adolescents in Southern Brazil: prevalence and associated factors. European Spine Journal, 21(7), 1234-1240. https://doi.org/10.1007/s00586-011-2056-3

Poussa, M. S., Heliövaara, M. M., Seitsamo, J. T., Könönen, M. H., Hurmerinta, K. A., \& Nissinen, M. J. (2005). Anthropometric measurements and growth as predictors of low-back pain: a cohort study of children followed up from the age of 11 to 22 years. European Spine Journal, 14(6), 595-598. https://doi.org/10.1007/s00586-004-0872-4

Ramadan, M. Z. (2011). Does Saudi school furniture meet ergonomics requirements?. Work, 38(2), 93-101. https://doi.org/ 10.3233/WOR-2011-1111

Ribaud, A., Tavares, I., Viollet, E., Julia, M., Hérisson, C., \& Dupeyron, A. (2013). Which physical activities and sports can be recommended to chronic low back pain patients after rehabilitation?. Annals of Physical and rehabilitation Medicine, 56(7), 576-594. https://doi.org/10.1016/j.rehab.2013.08.007

Salewytsch, A. J., \& Callaghan, J. P. (1999, October). Can quantified lumbar spine postures and trunk muscle activation levels predict discomfort during prolonged sitting. In Proceedings of the 31st Annual Conference of the Human Factors Association of Canada (pp. 316-321).

Salminen, J. J., Erkintalo, M., Laine, M., \& Pentti, J. (1995). Low Back Pain in the Young A Prospective ThreeYear Follow-up Study of Subjects With and Without Low Back Pain. Spine, 20(19), 2101-2107. https://doi.org/ 10.1097/00007632-199510000-00006 
Salminen, J. J., Oksanen, A., Mäki, P., Pentti, J., \& Kujala, U. M. (1993). Leisure time physical activity in the young. International journal of sports medicine, 14(07), 406-410. https://doi.org/10.1055/s-2007-1021200

Salminen, J. J., Pentti, J., \& Terho, P. (1992). Low back pain and disability in 14 - year - old schoolchildren. Acta Paediatrica, 81(12), 1035-1039. https://doi.org/10.1111/j.1651-2227.1992.tb12170.x

Sato, T., Ito, T., Hirano, T., Morita, O., Kikuchi, R., Endo, N., \& Tanabe, N. (2008). Low back pain in childhood and adolescence: a cross-sectional study in Niigata City. European Spine Journal, 17(11), 1441. https://doi.org/10.1007/s00586-008-0788-5.

Shan, Z., Deng, G., Li, J., Li, Y., Zhang, Y., \& Zhao, Q. (2013). Correlational analysis of neck/shoulder pain and low back pain with the use of digital products, physical activity and psychological status among adolescents in Shanghai. Plos one, 8(10), e78109. https://doi.org/10.1371/journal.pone.0078109

Silva, M. R., Badaró, A. F. V., \& Dall'Agnol, M. M. (2014). Low back pain in adolescent and associated factors: A cross sectional study with schoolchildren. Brazilian journal of physical therapy, 18(5), 402-409. https://doi.org/10.1590/bjpt-rbf.2014.0051

Torgén, M., \& Swerup, C. (2002). Individual factors and physical work load in relation to sensory thresholds in a middle-aged general population sample. European journal of applied physiology, 86(5), 418-427. https://doi.org/10.1007/s00421-001-0567-z.

Troussier, B., Davoine, P., De Gaudemaris, R., Fauconnier, J., \& Phelip, X. (1994). Back pain in school children. A study among 1178 pupils. Scandinavian journal of rehabilitation medicine, 26(3), 143-146.

Wedderkopp, N., Andersen, L. B., Froberg, K., \& Leboeuf-Yde, C. (2005). Back pain reporting in young girls appears to be puberty-related. BMC musculoskeletal disorders, 6(1), 52. https://doi.org/10.1186/1471-2474-6-52

Wilson, K. G., Eriksson, M. Y., Joyce, L. D., Mikail, S. F., \& Emery, P. C. (2002). Major depression and insomnia in chronic pain. The Clinical journal of pain, 18(2), 77-83. https://doi.org/10.1097/00002508-200203000-00002

Yue, P., Liu, F., \& Li, L. (2012). Neck/shoulder pain and low back pain among school teachers in China, prevalence and risk factors. BMC public health, 12(1), 789. https://doi.org/10.1186/1471-2458-12-789

\section{Copyrights}

Copyright for this article is retained by the author(s), with first publication rights granted to the journal.

This is an open-access article distributed under the terms and conditions of the Creative Commons Attribution license (http://creativecommons.org/licenses/by/4.0/). 